\title{
The high strain rate behaviour of polymers
}

\author{
N.N. Dioh, A. Ivankovic, P.S. Leevers and J.G. Williams \\ Imperial College of Science, Technology and Medicine, Department of Mechanical Engineering, Exhibition \\ Road, London SW7 2BX, U.K.
}

\begin{abstract}
Experimental results are presented for the strain rate sensitivity of polycarbonate in the strain rate range $10^{-4}$ to $10^{4} \mathrm{~s}^{-1}$, at $23^{\circ} \mathrm{C}$. The high strain rate tests are performed in a split Hopkinson pressure bar. Using thick specimens, there is an apparent increase in strain rate sensitivity in the high strain rate regime. This observed increase is significantly reduced when thinner specimens are employed suggesting that it does not represent inherent material behaviour. Numerical and analytical evidence is presented which shows that the apparent increase at high strain rates can be accounted for by stress wave propagation effects present in the test when high impact velocities are employed. The assumption of uniform stress and strain distribution within a split Hopkinson pressure bar specimen is therefore inappropriate at high impact velocities.
\end{abstract}

\section{INTRODUCTION}

There is a continuing debate as to the extent of the strain rate sensitivity of materials including polymers at high rates of strain. Some workers $(1,2,3)$, have reported an increase in the strain rate sensitivity of polymers and composites at high strain rates while similar observations have been made by other workers in metals $(4,5,6)$. As is evident in the literature, the true extent of the increase in strain rate sensitivity reported is inconsistent and depends on specimen dimensions and frictional constraints. In a recent paper $\left({ }^{7}\right.$ ) experimental stress/strain results by the present authors, are presented for four polymers tested in compression at $23^{\circ} \mathrm{C}$ between the strain rates of $10^{-4}-10^{4}$ $\mathrm{s}^{-1}$. The high strain rate results $\left(10^{2}\right.$ to $\left.10^{4} \mathrm{~s}^{-1}\right)$ were obtained in a SHPB system while the low and intermediate strain rate tests were carried out in an Instron and a Mayes testing machine respectively. The results show that the choice of specimen thickness at high strain rates significantly affects the measured flow stresses, with the thicker specimens showing an enhancement of flow stress at high rates. This apparent increase in strain rate sensitivity disappears completely for two of the polymers studied (medium density polyethylene and high density polyethylene) when thin specimens having a length to diameter ratio of 0.12 are used. Detailed studies $(8,9)$ have shown that the use of this length to diameter ratio will not lead to significant frictional effects in the specimen if a suitable lubricant such as petroleum jelly is applied between the specimen and the bars; otherwise an enhancement of flow stress for the wafer thin specimens will be obtained. Typical results of yield stress plotted against the logarithmic strain rate for polycarbonate Lexan are shown in figure 1. Clearly there is good agreement between the results at low and intermediate strain rates whereas at high strain rates thickness effects are 
observed. This good agreement at low and intermediate strain rates indicates that the discrepancy seen at high rates is not due to fundamental differences in the material properties.

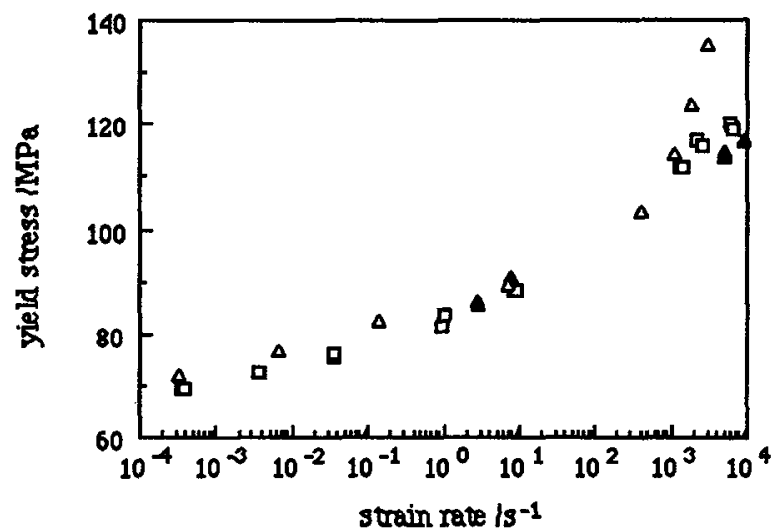

Figure 1. Measured yield stress versus logarithmic strain rate for $\mathrm{PC}$ at $23^{\circ} \mathrm{C}$ showing the effect of specimen thickness. Symbols: $\square, 4.4 \mathrm{~mm}$ thick specimens; $\triangle, 1.5 \mathrm{~mm}$ thick specimen; $\Delta, 8 \mathrm{~mm}$ thick specimen $\left({ }^{2}\right)$. The apparent increase in strain rate sensitivity at high strain rates is reduced when thinner specimens are used.

The literature on high rate testing indicates that the strain rate at which increased sensitivity is observed depends on specimen geometry $\left({ }^{6}\right)$, again suggesting that this observation may not represent inherent material properties. In this paper, analytical and numerical simulations of the split Hopkinson pressure bar (SHPB) technique are presented in order to determine its limitations and provide an explanation for the results of figure 1. This includes an investigation of the effect of impact velocity as this is thought to be the determining factor in whether or not large plastic wave fronts will be generated in the specimen; a phenomenon which will mean the classical split Hopkinson pressure bar analysis will be erroneous since stress equilibrium will not exist in the specimen.

\section{ANALYTICAL SIMULATION OF THE SHPB TEST}

For the ensuing analysis, a bi-linear rate independent model material behaviour has been chosen since analytical solutions modelling stress wave propagation within such a specimen are possible. The parameters for the material model used are presented in table 1 and have been approximated from experimental data for a typical polymer $(7,10)$. The bar properties are as specified by manufacturers.

Table 1. Material modelling parameters.

\begin{tabular}{|lccccc|}
\hline & $\begin{array}{c}\text { Elastic modulus, } \\
E / \mathrm{GPa}\end{array}$ & $S / \mathrm{MPa}$ & $\rho / \mathrm{kgm}^{-3}$ & $\begin{array}{c}\text { Yield stress, } \\
\sigma_{\mathrm{y}} / \mathrm{MPa}\end{array}$ & $\begin{array}{c}\text { Poisson's } \\
\text { ratio }\end{array}$ \\
\hline Material model & 1.7 & 136 & 950 & 25.2 & 0.40 \\
Aluminium bars & 73.1 & --- & 2796 & 410.0 & 0.33 \\
\hline
\end{tabular}

At high impact velocities, and for a loading pulse having a small rise time, the initial stress attained at the front face of the specimen will exceed $\sigma_{\mathrm{y}}$. This results in a plastic wave propagating towards the back face of the specimen, preceded by an elastic wave of magnitude $\sigma_{\mathrm{y}}(11)$. An analytical solution describing the plastic deformation of an ideal SHPB specimen under these conditions is presented below. It is assumed that the specimen is loaded by a Heaviside step function of infinite 
duration. Specimen unloading is therefore not considered. An analysis of a similar type has been carried out for a linear elastic specimen $\left({ }^{8}\right)$.

Assuming that the length of the loading pulse in the bar is much larger than the bar radius and that its propagation can be represented by the one-dimensional wave equation, expressions can be obtained for the variation of stress and velocity at the front and back faces of the SHPB specimen. The stress wave reflections which take place at the specimen boundaries after the incident pulse arrives at its front face are shown in figure 2 . In the figure, $C_{s}$ denotes the elastic wave speed in the specimen, given by $\sqrt{E / \rho}$, and $C_{\mathrm{p}}$ is the plastic wave speed given by $\sqrt{S / \rho}$. For a $z$-domain composed of a specimen of length, $l,(0 \leq z \leq l)$, the stresses and velocities can be denoted as $\sigma(z, t)$ and $v(z, t)$ at any time $t$. The initial boundary conditions for the system at $t=0$ may be taken as: $\sigma(z, t)=v(z, t)=0$ for all $z$. For $t>0, \sigma(z, t)$ and $v(z, t)$ will vary discontinuously as successive reflections take place in the specimen. The number of reflections which have taken place at $z$ from time zero may be represented by an integer, $n$ (figure 2 ).

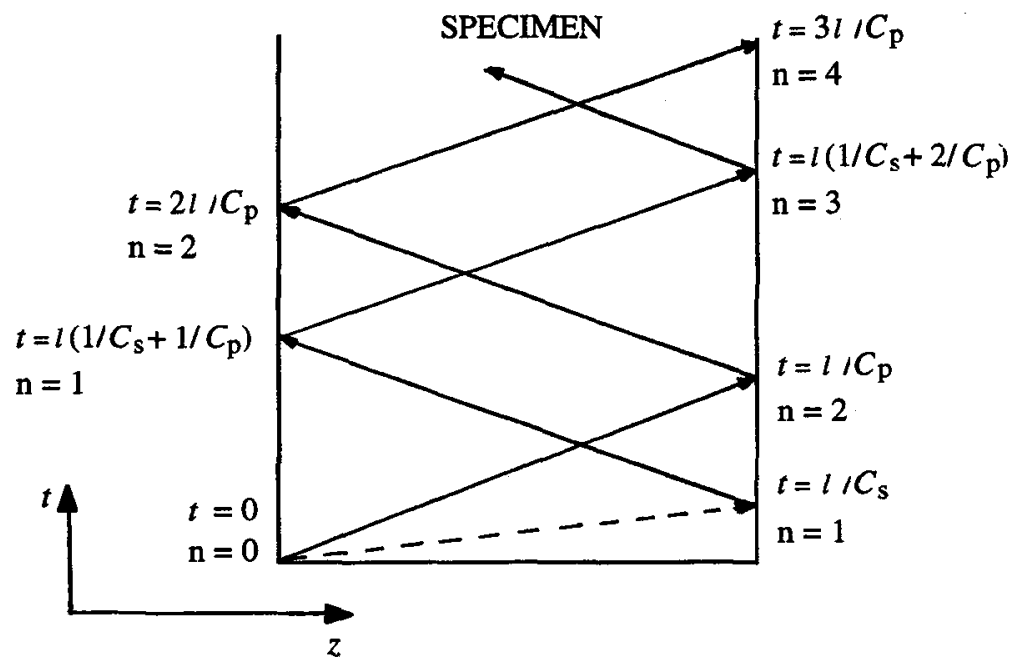

Figure 2. Location diagram showing stress wave interactions in the linear-elastic, linear-strain-hardening SHPB specimen at high impact velocities. The dotted line represents the preceding elastic wave.

For $z=0$ corresponding to the front face of the specimen, the variation of stress and velocity with time, $t$ take the form:

$$
\begin{aligned}
& \sigma(0, t)=\sigma_{\mathrm{I}}\left[1+R^{\mathrm{n}+1}\right]+\frac{\sigma_{\mathrm{y}}}{2}[1-R] R^{\mathrm{n}}\left[1-\frac{C_{\mathrm{p}}}{C_{\mathrm{s}}}\right] ; \quad \frac{\mathrm{n} l}{C_{\mathrm{p}}}<t<\frac{(\mathrm{n}+1) l}{C_{\mathrm{p}}}+\frac{l}{C_{\mathrm{s}}} \\
& v(0, t)=\frac{V}{2}\left[1-R^{\mathrm{n}+1}\right]-\frac{\sigma_{\mathrm{y}}}{2 Z_{\mathrm{b}}}[1-R] R^{\mathrm{n}}\left[1-\frac{C_{\mathrm{p}}}{C_{\mathrm{s}}}\right] ; \quad \frac{\mathrm{n} l}{C_{\mathrm{p}}}<t<\frac{(\mathrm{n}+1) l}{C_{\mathrm{p}}}+\frac{l}{C_{\mathrm{s}}}
\end{aligned}
$$

for $n=0,2,4,6, \ldots$ and similarly for $n=1,3,5,7, \ldots$ In the same way, expressions can be obtained for the back face of the specimen (10). In equations (1) and (2), $R=\frac{Z_{\mathrm{p}}-Z_{\mathrm{b}}}{Z_{\mathrm{p}}+Z_{\mathrm{b}}}$ is the reflection coefficient of the bar-specimen where $Z_{\mathrm{p}}$ represents the mechanical impedance $\rho C_{\mathrm{p}}$ of the 
specimen after yield. $\sigma_{\mathrm{I}}=\frac{Z_{\mathrm{b}} V}{2}$ is the magnitude of the incident pulse, where $V$ is the impact velocity of the projectile.

Hence the stresses and velocities at the specimen faces during deformation can be obtained for all $t$. Using the above analysis, reconstituted stress strain curves at typical impact velocities employed in the experiments have been computed and the results are shown in figure 3 in terms of $5 \%$ flow stress against logarithmic strain rate.

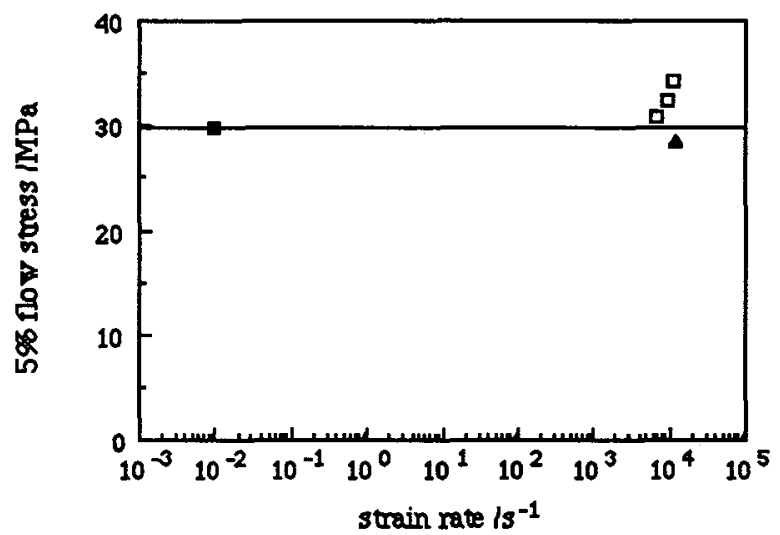

Figure 3. Computed 5\% flow stress versus logarithmic strain rate for the rate independent bi-linear model showing the effect of specimen thickness. Symbols: $\square, 4.4 \mathrm{~mm}$ thick specimens; $\triangle, 1.5 \mathrm{~mm}$ thick specimen. The solid line represents the true flow stress at $5 \%$ strain of the model material.

The apparent increase in strain rate sensitivity at high strain rates arises purely as a result of stress wave propagation effects in the specimen. The trend exhibited in figure 3 is indeed the same as that seen from numerous experiments, most of which have so far been accepted as representing the intrinsic behaviour of the materials. It is clear that this apparent increase in strain rate sensitivity can also be explained solely in terms of stress wave propagation effects using a simple 1-D analytical solution.

\section{NUMERICAL SIMULATION OF THE SHPB TEST}

The finite volume numerical code used in simulating the SHPB test is based on the solution of the equation of motion for a solid body of volume $V$, bounded by a surface $S(12,13)$. It is a 3-D code capable of transient non-linear analysis. The discretisation scheme used together with the right boundary conditions for a given problem results in a system of linear equations which are temporarily decoupled and solved sequentially by a line by line Tri - Diagonal Matrix Algorithm.

In the present work, the complete SHPB system is modelled, including both bars and the specimen. The properties of the bars and the specimen are those in table 1 . The bars are of diameter $15.8 \mathrm{~mm}$ and length $1200 \mathrm{~mm}$. The projectile is of length $200 \mathrm{~mm}$ and is of the same diameter and material as the bars. Care was taken to ensure that the dimensions of the real geometry was accurately represented; e.g. dimensions of the specimen and the bars and the position of the strain gauges. A uniform mesh was used to avoid generation of the spurious wave reflections within the code. Numerical simulations at impact velocities of 12 and $30 \mathrm{~m} \mathrm{~s}^{-1}$ have been carried out for the specimen material model presented in table 1 . The loading pulse corresponding to each impact velocity is applied in terms of input displacements at the impact end of the incident bar. The 
loading pulse had a rise time of $15 \mu \mathrm{s}$, a value which corresponds to typical rise times encountered for SHPB compression tests in practice.

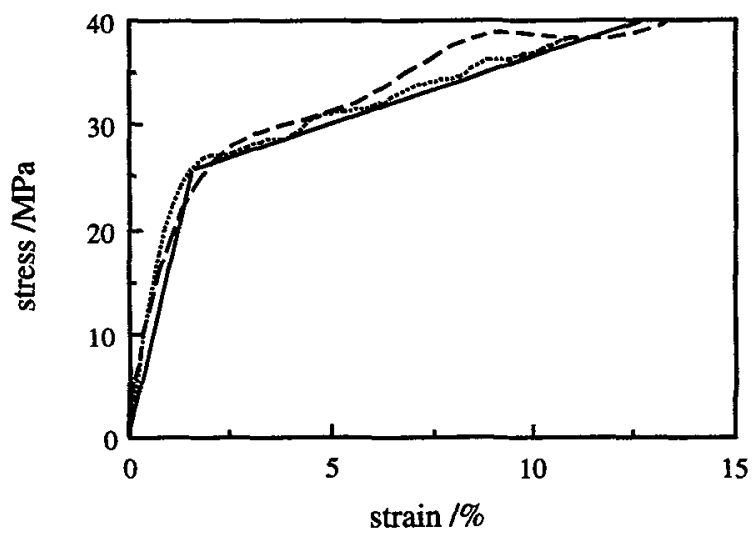

Figure 4. Numerically determined stress strain curve at impact velocities of 12 and $30 \mathrm{~m} \mathrm{~s}^{-1}$ superimposed over the input stress strain curve. Input bi-liear material model, $-; 12 \mathrm{~m} \mathrm{~s}^{-1}, \ldots, \ldots 30 \mathrm{~m} \mathrm{~s}^{-1},-\cdots$.

The numerically determined stress strain curves at 12 and $30 \mathrm{~ms}^{-1}$ are shown in figure 4 . These are computed from the reflected and transmitted pulses measured at the strain gauge stations by using the classical SHPB analysis assuming that no stress and strain non-uniformities exist within the specimen $\left({ }^{14}\right)$. The average strain rates are $1.5 \times 10^{3}$ and $5 \times 10^{3} \mathrm{~s}^{-1}$ respectively for the $12 \mathrm{~ms}^{-1}$ and $30 \mathrm{~ms}^{-1}$ impact velocities. Also shown in figure 4 is the input bi-linear stress strain curve. At the lower impact velocity of $12 \mathrm{~m} \mathrm{~s}^{-1}$, there is good agreement between the numerically determined stress strain curve and the input curve. Significant errors, however, arise when an impact velocity of $30 \mathrm{~m} \mathrm{~s}^{-1}$ is employed. The discrepancy at the higher strain rate is therefore due solely to the increase in impact velocity as it is the only parameter that is altered between the two test cases.

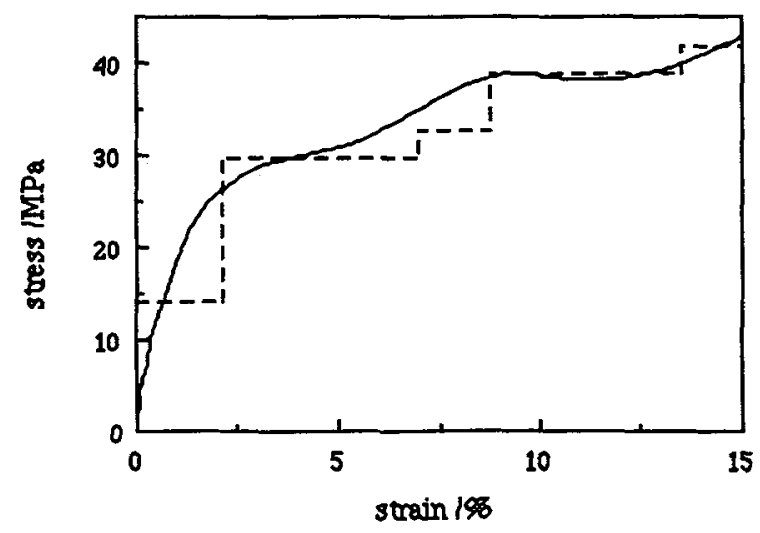

Figure 5. Comparison between the analytical and numerical stress strain curves at an impact velocity of $30 \mathrm{~m} \mathrm{~s}^{-1}$ for the input bi-linear material model depicted in figure 4. Numerical, ——; Analytical , - - - 


\section{DISCUSSION}

A comparison between the analytical and numerical simulations for an impact velocity of $30 \mathrm{~m} \mathrm{~s}^{-1}$ is presented in figure 5. It is noted that the steps in the analytical stress strain curve, corresponding to the propagation of plastic wave fronts in the specimen, are fairly well reproduced in the numerical curve. Clearly a rise time of $15 \mu \mathrm{s}$ as is encountered in practice is sufficiently small for stress wave propagation effects to be important at high strain rates. The results presented in figure 6 indicate, therefore, that the increase in flow stress seen in the reconstituted stress strain curve at $30 \mathrm{~m} \mathrm{~s}^{-1}$ is due to the propagation of large plastic wave fronts within the specimen which are inevitable at high impact velocities. For the materials studied, the fundamental assumptions in the classical SHPB theory are therefore invalid at high impact velocities. This will also be expected for other materials exhibiting similar plastic wave speeds. Due to the difficulty of modelling the exact material behaviour (which is the unknown) the only viable method of determining whether or not high strain rate measurements are affected by stress wave propagation effects will be to test various specimen thicknesses and show the values at the same strain rates to be the same. It is now clear why thinner specimens should yield more accurate results: lower impact velocities are employed for the same strain rates, thereby leading to smaller stress and strain gradients in the specimen.

\section{CONCLUSION}

Analytical and numerical simulations show that the use of very high impact velocities in split Hopkinson pressure bar tests will lead to the manifestation of large stress and strain gradients within the specimen associated with the propagation of plastic wave fronts within its boundaries. The effect of this is an enhancement in measured flow stresses at high strain rates, of a kind which has previously been interpreted as an inherent increase in strain rate sensitivity. Assuming that stress wave propagation effects are absent in the SHPB test, as is the case in the classical analysis, is therefore inappropriate at high impact velocities for the materials studied.

\section{ACKNOWLEDGEMENTS}

The authors are grateful to the Quantum Chemical Corporation for providing the financial support necessary during the course of the work. We would also like to thank ICI Plastics for providing the polymer samples.

\section{REFERENCES}

[1] Chou, S. C., Robertson, K. D. and Rainey, J. H., Exp. Mech. 13 (1973) 422

[2] Rietsch, F. and Bouette, B., Eur. Polym. J. 26 (1990) 1071

[3] Harding, J., in Materials at high strain rates, Ed. Blazynski, T. Z. Elsevier applied Science.(1987) 133.

[4] Zukas, J. A., Nicholas, T., Swift, H. S., Greszczuk, L. B. and Curran, D.R., 1982 Impact Dynamics. John Wiley and Sons.

[5] Parry, D.J. and Walker, A.G., Mech. Prop. at High Rates of Strain, (ed. J. Harding), The Instit. Phys. Bristol and London. (1989) 329.

[6] Gorham, D.A., 3rd Inter. Conf. on Mech. and Phys. Behaviour of Mat. under dyn. loading, Strasbourg. Journal de Physique IV, Colloque C3, suppl. au Journal de Physique III, 1 (1991) 411.

[7] Dioh, N. N., Leevers, P. S. and Williams, J. G., Polymer 34 (1993) 4230

[8] Briscoe, B. J. and Nosker, R.W., Wear 95 (1984) 241

[9] Walley, S. M., Field, J. E., Hope, P.H. and Safford, N. A., Phil. Trans. Royal. Soc. London A328 (1989) 1

[10] Dioh, N. N.,PhD Thesis, Department of Mechanical Engineering, Imperial College. (1993)

[11] Johnson, W., Impact Strength of Materials. Arnold. (1972) 225

[12] Ivankovic, A, Demirdzic, I., Williams, J. G. and Leevers, P. S., Int. J. Fracture. 66 (1994) 357

[13] Ivankovic, A. and Dioh, N. N., Conference on Computational mechanics in the UK.Manchester (1994) 83.

[14] Lindholm, U.S.,J. Mech. Phys. Solids 12 (1964) 317 